Das Arztgeheimnis ist wie eine Uhr mit Komplikationen: Die Komplexität steigert zwar den Wert, erschwert aber auch die Anwendung. Die besorgten Äusserungen über die Gefährlichkeit bestimmter Häftlinge und die angebliche Blindheit der Ärzte haben die forensischen Psychiater unter Druck gesetzt. Die zutage getretenen Divergenzen haben allerdings nichts mit dem Röstigraben zu tun, wie der untenstehende Artikel zeigt. Die Rechtslage ist nämlich eindeutig; bloss trauen die Ärzte sich nicht, das Potential des Arztgeheimnisses - letztendlich auch zum Vorteil der Gesellschaft - voll auszuschöpfen.

Monique Gauthey, Fachärztin, Mitglied des Zentralvorstandes der FMH, Departementsverantwortliche Spitalärztinnen und -ärzte

\title{
Das Arztgeheimnis im Gefängnis: eine falsche Polemik
}

\author{
Dominique Sprumont ${ }^{\mathrm{a}}$, Philippe Ducor \\ a Prof. Dr. iur., Stv. Direktor des Instituts für Gesundheitsrecht, Vize-Direktor SSPH+ / ERSP, Universität Neuenburg \\ ${ }^{b}$ Prof. Dr. iur. et med., Rechtsanwalt, Genf
}

Unter dem Titel Mythen und Realitäten haben Steffen Lau und Josef Sachs die Frage des Arztgeheimnisses bei der Behandlung gefährlicher Häftlinge neu aufgeworfen [1]. Ihre Absicht war, als Antwort auf die nach ihrer Meinung in der Westschweiz vorherrschende «emotionale» Tonalität, die Diskussion auf die wirklichen Gegebenheiten der forensischen Psychiatrie zurückzuführen. Leider wirkt ihr Artikel der Mythenbildung nicht entgegen, sondern verstärkt diese sogar, indem er den rechtlichen Rahmen der ärztlichen Tätigkeit im Gefängnis eher frei auslegt.

Zunächst soll an den wesentlichen Rechtsgrundsatz erinnert werden, nach dem in Ermangelung einer spezifischen Rechtsvorschrift die allgemeinen Vorschriften anwendbar sind. Konkret bedeutet dies, dass Ärztinnen und Ärzte, die im Gefängnis oder im Auftrag der Justizbehörden tätig sind, auf inhaftierte Patienten dieselben Grundregeln anzuwenden haben wie auf nicht inhaftierte, sofern keine gesetzlichen Bestimmungen existieren, die ihre Rechte und Pflichten anderweitig regeln. Lau und Sachs vertreten die Auffassung, das spezielle Rechtsverhältnis zwischen dem gefährlichen Häftling und der Vollzugs- und Justizbehörde liefere einen ausreichenden Rechtfertigungsgrund. Da die Beziehung zwischen Patient und Arzt im Gefängnis nicht unter das Privatrecht falle, unterlägen Patientenrechte und berufliche Pflichten vollkommen anderen Regeln. Dieses Argument stimmt nachdenklich: Wörtlich genommen würde es bedeuten, dass in öffentlichen Einrichtungen hospitalisierte und somit nicht dem Privatrecht unterstehende Patienten ebenfalls einem speziellen Rechtssystem unterworfen sind. Dies ist jedoch weit gefehlt. Es genügt, Art. 40 des Medizinalberufegesetzes (MedBG) $\mathrm{zu}$ konsultieren sowie die zahlreichen kantonalen Rechtsvorschriften im Gesundheitswesen, welche Ärztinnen und Ärzte zur Einhaltung der Patientenrechte im öffentlichen genauso wie im privaten Bereich verpflichten. Der Arzt bleibt jederzeit Arzt. Dieser Grundsatz wird im Übrigen durch kantonale Ausführungsgesetze zum Strafgesetzbuch bestätigt, die anerkennen, dass in Bezug auf die Gesundheit Häftlinge dieselben Rechte besitzen wie alle anderen Personen. Die FMH hat von daher stets den Grundsatz der Gleichwertigkeit der Behandlung in der Gefängnismedizin oder auch, wie erst jüngst wieder, im Rahmen der zwangsweisen Rückführungen verteidigt.

Lau und Sachs kommen ausserdem zu dem Schluss, dass nicht nur das Recht der Häftlinge auf Schutz ihrer Privatsphäre zähle, sondern auch dasjenige der Bevölkerung auf Sicherheit. Dieses Argument erinnert an den Slogan «Im Zweifel für die Gesellschaft», den gewisse Politikerinnen und Politiker in Mordfällen vertraten. Einer so allgemein gefassten Aussage lässt sich schwer widersprechen. Die Realität ist jedoch komplexer. Die Frage dreht sich weniger um die Rechte der gefährlichen 
Häftlinge als vielmehr um den Auftrag, den die Justiz den Ärzten kraft des Schweizerischen Strafgesetzbuchs (StGB) erteilt. Zur Erinnerung: Gerade weil Straftäterinnen und Straftäter gefährlich sind, ordnen die Richter therapeutische Massnahmen an [2], um ihre Gefährlichkeit zu verringern. Der Richter kann sich dieser Pflicht nicht entziehen. Forensische Psychiater, die im Auftrag der Justiz tätig werden, sind somit unter Berücksichtigung ihrer ärztlichen Pflichten von Rechts wegen der Gesellschaft verpflichtet. Genau aufgrund dieses Auftrags haben zahlreiche Gefängnisärztinnen und -ärzte, die kantonalen Ärztegesellschaften, die FMH und die Schweizerische Akademie der Medizinischen Wissenschaften das Arztgeheimnis im Gefängnis stets verteidigt und werden dies auch weiterhin tun. Der forensische Psychiater kann seine Mission ohne das Arztgeheimnis nicht erfüllen.

Die Wirksamkeit einer Behandlung ist in hohem Grad abhängig vom Vertrauensverhältnis, das zwischen Arzt und Patient entsteht. Das Arztgeheimnis [3] ist der Eckpfeiler dieses Vertrauensverhältnisses. Dieses zu gefährden würde bedeuten, den Therapeuten in seinen Behandlungsmöglichkeiten einzuschränken und damit dem im Strafgesetzbuch vorgesehenen therapeutischen Auftrag seinen Sinn zu nehmen. Dies heisst nicht, dass Ärztinnen und Ärzte sich nicht mit den Vollzugs- und Justizbehörden austauschen dürfen. Im Gegenteil: Wie Lau und Sachs zu Recht feststellen, wird jede therapeutische Massnahme durch die zuständige Strafvollzugsbehörde überwacht. Der zwischen dem inhaftierten Patienten, dem Arzt und der Justiz festgelegte «Behandlungsauftrag» präzisiert - in voller Kenntnis und mit vollem Einverständnis des Häftlings - die Elemente, über die ein Informationsaustausch zwischen Arzt und Justiz stattfindet, wie beispielsweise die Einhaltung der ärztlichen Verordnungen, die Teilnahme an den Sitzungen, der Behandlungsfortschritt gemäss den Zielen zur Verringerung der Gefährlichkeit usw. Die entsprechenden Berichte werden regelmässig aufgrund eines zuvor erstellten Zeitplans erstellt. Diese Aspekte sind unbestritten und integraler Bestandteil der ärztlichen Tätigkeit in Schweizer Gefängnissen.

Die durch die Änderung verschiedener Ausführungsgesetze des Strafgesetzbuchs in der Westschweiz aufgeworfene Frage ist eine andere. Sie betrifft die Fakten und Vorkommnisse ausserhalb des Behandlungsauftrags, die einen Einfluss auf die Einschätzung der Gefährlichkeit des Häftlings haben könnten. Diesbezüglich sei an den grundlegenden Unterschied zwischen der Therapeuten- und der Expertenrolle erinnert, die forensische Psychiaterinnen und Psychiater übernehmen müssen. Die Strafprozessordnung (StPO) unterscheidet ganz klar zwischen beiden Rollen und legt fest, dass ein Experte nicht gleichzeitig als Therapeut tätig sein kann und umgekehrt [4]. Dieser allgemeine Rechtsgrundsatz findet sich auch in den entsprechenden Ausstandsregeln der Zivilprozessordnung [5] und im Verwaltungsverfahrensgesetz [6]. Der Grund liegt auf der Hand. Der Therapeut steht in enger Beziehung zu seinem Patienten, was seine Einschätzungen beeinflussen kann und Quelle eines Interessenkonflikts ist. Zum Schutz der Gesellschaft wurden daher im Strafgesetzbuch spezielle Verfahren zur Beurteilung der Gefährlichkeit von Straftätern mit besonders hohem Risiko festgelegt [7]. Diese Beurteilung erfolgt unter richterlicher Aufsicht und unter Beteiligung mehrerer Expertinnen und Experten, meistens in einem interdisziplinären Rahmen. In Bezug auf die ärztliche Weitergabe von Informationen an die Justiz im Rahmen eines Strafverfahrens wird auf Artikel 171 Absatz 3 StPO hingewiesen, der das Arztgeheimnis ausdrücklich schützt. Das Bundesgericht hat deshalb das Basler Gesundheitsgesetz, das eine allgemeine Informationspflicht für solche Sachverhalte schaffen wollte, als dieser Bestimmung zuwiderlaufend beurteilt [8].

Es ist bedauerlich, dass Lau und Sachs diesen Unterschied zwischen der Therapeuten- und der Expertenrolle nicht stärker berücksichtigen. Ihr Artikel vermittelt den Eindruck, dass die Ärzte, die ihrer Therapeutenrolle gewissenhaft nachkommen, ihre Pflicht der Gesellschaft gegenüber vernachlässigen. Die Autoren scheinen die Auffassung zu vertreten, dass die Gefängnisärztinnen und -ärzte zuerst als Hilfskräfte der Justiz und erst in zweiter Linie als Therapeuten fungieren sollen. Dies ist eine beunruhigende Vorstellung, die den Zielen der Strafprozessordnung und der Strafverfolgungspolitik zuwiderläuft. In ihrer karikierenden Darstellung der Gefängnisärzte, die auf die Wahrung des Arztgeheimnisses bedacht sind und ihren Patienten vorgeblich zu nahe stehen, vergessen Lau und Sachs zu erwähnen, dass sich in der Praxis genau diese Ärztinnen und Ärzte auf Basis ihres Behandlungsauftrags regelmässig mit der Justiz und den Vollzugsbehörden austauschen. Ebenso scheinen sie zu ignorieren, dass ebendiese Ärzte laut Artikel 17 StGB im Falle einer unmittelbaren Gefahr die Behörden unverzüglich in Kenntnis zu setzen haben. In der Medizin gibt es keine alleinige Wahrheit. Es ist wichtig, dass die praktizierenden Ärztinnen und Ärzte unterschiedliche Ansätze verteidigen können. Allerdings gilt für alle derselbe rechtliche Rahmen, und es ist sinnvoll, sich mitunter an seine Inhalte zu erinnern. Die FMH und die Konferenz Schweizerischer Gefängnisärzte (KSG) sind die richtigen Institutionen für diese Aufgabe! Auf dieser Grundlage können die Fachleute sich positionieren, indem sie geeignete Praktiken beschreiben, statt sich in irreführende Polemik zu verlieren. 